\title{
The Resolution of the Anti-Pasch Conjecture
}

\author{
M.J. Grannell and T.S. Griggs \\ Department of Pure Mathematics, The Open University, \\ Milton Keynes MK7 6AA, UK \\ C.A. Whitehead \\ Department of Mathematical and Computing Sciences, \\ Goldsmiths College, London SE14 6NW, UK
}

This is a preprint of an article published in the Journal of Combinatorial Designs, 8, 2000, p300-309, (C)2000 (copyright owner as specified in the journal).

\begin{abstract}
We show that an anti-Pasch Steiner triple system of order $v$ exists for $v \equiv 1$ or $3(\bmod 6)$, apart from $v=7$ and 13 .
\end{abstract}


Running Head: Anti-Pasch STS

AMS Classification: 05B07

Keywords: Steiner triple system, Pasch configuration, quadrilateral

Corresponding author: M.J. Grannell

Home address: 17 Royal Avenue, Leyland, Lancashire PR25 1BQ, UK.

Telephone: (+44) 01772-433430, email: m.j.grannell@open.ac.uk 


\section{Introduction}

A Steiner triple system $S=(V, \mathcal{B})$ of order $v$, denoted by $\operatorname{STS}(v)$, is a collection $\mathcal{B}$ of triples (3-element subsets) of the set $V$, where $|V|=v$, such that each unordered pair of elements (points) of $V$ is contained in precisely one triple from $\mathcal{B}$. It is well known that an $\operatorname{STS}(v)$ exists if and only if $v \equiv 1$ or $3(\bmod 6)$; such values of $v$ are called admissible.

A Pasch configuration, also known as a quadrilateral, consists of four triples of a Steiner triple system whose union is a set of six points, that is to say, four triples which must be of the form $\{a, b, c\},\{a, y, z\},\{x, b, z\}$ and $\{x, y, c\}$. An $\operatorname{STS}(v)$ is anti-Pasch or quadrilateral-free if it does not contain a Pasch configuration. We will denote such a system by $\operatorname{QFSTS}(v)$. It is a long standing conjecture that for all admissible $v$, apart from $v=7$ and $v=13$, there exists a QFSTS $(v)$. The problem goes back to Erdös [4] and Brouwer [1], and is of some practical significance in connection with disk data storage arrays (see [3]).

The situation for small values of $v$ is as follows. Up to isomorphism, the STS(7) is unique and contains seven Pasch configurations. The unique STS(9) is anti-Pasch. There are two non-isomorphic STS(13)s, one of which contains thirteen Pasch configurations and the other eight. Of the 80 nonisomorphic STS(15)s, precisely one has no Pasch configuration; this is \#80 in the standard listing given in [10]. For larger values of $v$, a complete enumeration of $\operatorname{STS}(v)$ is not feasible. Stinson and Ferch [14] constructed over $2 \times 10^{6}$ non-isomorphic STS(19)s by hill-climbing and it is estimated that there are in the region of $10^{9}$ non-isomorphic systems. Murphy [12] has constructed over $10^{3}$ non-isomorphic QFSTS(19)s. For each admissible $v<100, v \neq 7$ or 13 , a $\operatorname{QFSTS}(v)$ is known to exist [2].

It was shown $[1,6]$ that for every $v \equiv 3(\bmod 6)$, a $\operatorname{QFSTS}(v)$ exists. More recently Ling, Colbourn and two of the present authors [9] substantially narrowed the spectrum of possible exceptions in the case $v \equiv 1(\bmod 6)$ by showing, inter-alia, that exceptions must lie in the residue classes 13, 31 and $67(\bmod 72)$. In fact, even within these residue classes, it is possible to deal with some values. However, the range of possibilities left open by [9] apparently remains infinite.

The purpose of the current paper is to present a new construction for QFSTS $(v)$. This relies for its application on the construction of certain auxiliary designs. With the aid of this construction and appropriate auxiliary designs, the conjecture is established. The construction itself is presented in 
Section 2, where the auxiliary designs are also defined. Applications of the construction are given in Section 3. One of the auxiliary designs is listed explicitly in Section 4. This is the one necessary for dealing with the residue class 31 modulo 72. Space considerations preclude a complete listing of all the auxiliary designs required because some of these are quite large. A full listing of all the auxiliary designs needed to complete the proof is available from the Journal of Combinatorial Designs (JCD) website [15].

A group divisible design $(\mathrm{GDD})$ is a triple $(X, \mathcal{G}, \mathcal{B})$ which satisfies the following properties:

1. $\mathcal{G}$ is a partition of the set $X$ (of points) into subsets called groups;

2. $\mathcal{B}$ is a collection of subsets of $X$ (called blocks) such that every pair of points from distinct groups occurs in a unique block, and no pair of points from a single group occurs in any block.

The group type (type) of the GDD is the multiset $\{|G|: G \in \mathcal{G}\}$ and is usually described using an "exponential" notation. Thus, a group type $1^{i} 2^{j} 3^{k} \ldots$ denotes $i$ groups of size $1, j$ groups of size $2, k$ groups of size 3 , etc. If $K$ is a set of positive integers, each of which is greater than or equal to 2 , then we say that a $\operatorname{GDD}(X, \mathcal{G}, \mathcal{B})$ is a $K$-GDD if $|B| \in K$ for every block $B \in \mathcal{B}$. When $K=\{k\}$ we simply write $k$ for $K$. A $K$-GDD is said to be uniform if all the groups have the same size, that is, if it is of type $g^{u}$. A transversal design $T D(k, n)$ is a $k$-GDD of type $n^{k}$.

A $T D(3, n)$ is equivalent to an $n \times n$ Latin square. A subsquare of a Latin square is a square subarray that is itself a Latin square. A Latin square is an $N_{2}$-Latin square if it contains no subsquare of order 2. An $N_{2}$-Latin square of order $n$ (i.e. an $n \times n$ square) exists for all $n \geq 3$ and $n \neq 4[7,8,11]$. We shall refer to a $T D(3, n)$ without any sub- $T D(3,2)$ as an $N_{2}-T D(3, n)$.

\section{The Construction}

As previously remarked, the construction employs auxiliary designs. These designs are now described. Section 4 gives several specific instances, demonstrating that the class of such designs is far from empty.

$\operatorname{An} \operatorname{STS}(u,-m)$ is a triple $(U, M, \mathcal{B})$, where $U$ is a set of points having cardinality $u, M \subseteq U$ has cardinality $m$, and $\mathcal{B}$ is a collection of triples of points with the property that every pair of points $\{\alpha, \beta\}$, with $\alpha \in U, \beta \in U \backslash M$ 
appears in precisely one triple from $\mathcal{B}$, and no pairs $\{\alpha, \beta\}$ with $\alpha, \beta \in M$ appear in any triple from $\mathcal{B}$. For $u$ and $m$ both admissible (the only cases we will consider here), an $\operatorname{STS}(u,-m)$ is an $\operatorname{STS}(u)$ with an $\operatorname{STS}(m)$ subsystem removed. A $\operatorname{QFSTS}(u,-m)$ is an $\operatorname{STS}(u,-m)$ containing no Pasch configurations. An $\operatorname{STS}(u,-m)$ is said to be $m$-bipartite if the points of $U \backslash M$ can be partitioned into two classes $A$ and $B$, each of cardinality $n$ (so that $u=m+2 n$ ), in such a way that no triples of the design are labelled $M, A, A$ or $M, B, B$. An $m$-bipartite $\operatorname{QFSTS}(u,-m)$ will be denoted by $\operatorname{BQFSTS}(u,-m)$.

It is easily seen that for admissible $u=m+2 n$, an $m$-bipartite $\operatorname{STS}(u,-m)$, can only exist if $m \leq n$.

Theorem 2.1 Suppose that there exists a QFSTS $(2 n+m)$ and a BQF$S T S(2 n+m,-m)$, where $n=3$ or $n \geq 5$. Suppose also that there exists a $\operatorname{QFSTS}(v)$. Then there exists a $\operatorname{QFSTS}(n(v-1)+m)$.

Proof. Take a $\operatorname{QFSTS}(v)$ and delete a point, say $\infty$, to obtain a 3-GDD of type $2^{(v-1) / 2}$ with groups $\left\{a_{i}, b_{i}\right\}$ for $i=1,2, \ldots,(v-1) / 2$. Replace each point $a_{i}$ by $n$ points $a_{i}^{1}, a_{i}^{2}, \ldots, a_{i}^{n}$, and do likewise for each $b_{i}$. Then use an $N_{2}-T D(3, n)$ to produce a 3-GDD of type $(2 n)^{(v-1) / 2}$. Denote the groups of this design by $G_{1}, G_{2}, \ldots, G_{(v-1) / 2}$, so that $G_{i}=A_{i} \cup B_{i}$, where $A_{i}=\left\{a_{i}^{1}, a_{i}^{2}, \ldots, a_{i}^{n}\right\}$ and $B_{i}=\left\{b_{i}^{1}, b_{i}^{2}, \ldots, b_{i}^{n}\right\}$. Next, take $m$ new points to form a set $M=\left\{\infty_{1}, \infty_{2}, \ldots, \infty_{m}\right\}$, say. Place a copy of the $\operatorname{QFSTS}(2 n+m)$ onto the points of $M \cup A_{1} \cup B_{1}$. Then, for each $i=2,3, \ldots,(v-1) / 2$, place a copy of the BQFSTS $(2 n+m,-m)$ onto the points of $M \cup A_{i} \cup B_{i}$ so that the labelling partition corresponds to $M, A_{i}$ and $B_{i}$. Call the triples of the 3GDD of type $(2 n)^{(v-1) / 2}$ vertical triples and the triples of the QFSTS $(2 n+m)$ and the BQFSTS $(2 n+m,-m)$ horizontal triples. Points and triples from the same copy of the BQFSTS $(2 n+m,-m)$ will be said to be on the same level; those from the QFSTS $(2 n+m)$ will be said to be from the top level. We will refer to a point $x$ of the 3 -GDD of type $2^{(v-1) / 2}$ as being the projection of any one of the $n$ points $x^{1}, x^{2}, \ldots, x^{n}$ which replaced it in forming the 3-GDD of type $(2 n)^{(v-1) / 2}$.

We prove firstly that the resulting set of triples, say $\mathcal{B}$, forms an $\operatorname{STS}(n(v-$ 1) $+m)$ on the set of points $U=\left(\bigcup_{i=1}^{(v-1) / 2} G_{i}\right) \cup M$.

To do this, consider first any pair of points from $U$ which come from the same level. If both these points lie in $M$, then they are contained in a triple of the $\operatorname{QFSTS}(2 n+m)$. Otherwise, at least one the two points lies in $G_{i}$ for 
some value of $i$, say $i=i^{*}$. The other point will lie in $G_{i^{*}}$ or in $M$. If $i^{*}=1$, the pair is contained in a triple of the $\operatorname{QFSTS}(2 n+m)$; if $i^{*}>1$, then the pair is contained in a triple of the appropriate $\operatorname{BQFSTS}(2 n+m,-m)$.

Next consider any pair of points that come from different levels. In this case, neither point lies in $M$, so suppose that the points are $x_{i}^{p}$ and $y_{j}^{q}$, where $i \neq j$. The projections $x_{i}$ and $y_{j}$ lie in a block of the 3-GDD of type $2^{(v-1) / 2}$ and so $\left\{x_{i}^{p}, y_{j}^{q}\right\}$ will be contained in a triple of type $(2 n)^{(v-1) / 2}$. It follows that the triples of $\mathcal{B}$ cover all pairs from $U$.

To complete the proof that $(U, \mathcal{B})$ forms a Steiner triple system, it now suffices to show that $|\mathcal{B}|=(n(v-1)+m)(n(v-1)+m-1) / 6$. Note that the number of horizontal triples is

$$
\frac{(2 n+m)(2 n+m-1)}{6}+\left(\frac{v-3}{2}\right)\left(\frac{(2 n+m)(2 n+m-1)}{6}-\frac{m(m-1)}{6}\right)
$$

and the number of vertical triples is

$$
(n(v-1))(n(v-1)-2 n) / 6 .
$$

Addition gives the total number of triples as $(n(v-1)+m)(n(v-1)+m-1) / 6$, as required.

It remains to establish that the design is anti-Pasch. Suppose to the contrary that a Pasch configuration $P$ is present.

Assume, initially, that $\infty_{x}$ is a point of $P$. Then $P$ contains at least two horizontal triples. If these two triples were from the same level, then $P$ would be from that level, contradicting the assumption that the $\operatorname{STS}(2 n+m)$ and the $\operatorname{STS}(2 n+m,-m)$ are anti-Pasch. Thus the two triples containing $\infty_{x}$ must be from different levels. If neither of these two levels is the top level, then the two blocks would necessarily have the form $\left\{\infty_{x}, a_{p}^{h}, b_{p}^{i}\right\}$ and $\left\{\infty_{x}, a_{q}^{j}, b_{q}^{k}\right\}$, where $p$ and $q$ denote the levels, and $P$ must therefore contain two vertical triples. But then projecting these two vertical triples back to the original QFSTS $(v)$ would form a Pasch configuration with the triples $\left\{\infty, a_{p}, b_{p}\right\}$ and $\left\{\infty, a_{q}, b_{q}\right\}$, again a contradiction. There remains the possibility that one of the two horizontal triples comes from the top level: if this triple is of the form $\left\{\infty_{x}, a_{1}^{h}, b_{1}^{i}\right\}$, then the argument leading to a contradiction is the same as for levels $p, q>1$. Otherwise, the top triple has one of the forms $\left\{\infty_{x}, a_{1}^{h}, a_{1}^{i}\right\},\left\{\infty_{x}, b_{1}^{h}, b_{1}^{i}\right\},\left\{\infty_{x}, \infty_{y}, a_{1}^{h}\right\},\left\{\infty_{x}, \infty_{y}, b_{1}^{h}\right\}$ or $\left\{\infty_{x}, \infty_{y}, \infty_{z}\right\}$. 
In the first of these five cases, the two horizontal triples may be taken as $\left\{\infty_{x}, a_{1}^{h}, a_{1}^{i}\right\}$ and $\left\{\infty_{x}, a_{q}^{j}, b_{q}^{k}\right\}$, where $q>1$. The third and fourth triples then have the forms $\left\{a_{1}^{h}, a_{q}^{j}, w_{r}^{l}\right\}$ and $\left\{a_{1}^{i}, b_{q}^{k}, w_{r}^{l}\right\}$ for some $r \neq 1, q$. Projecting back to the $\operatorname{QFSTS}(v)$, we then have triples $\left\{a_{1}, a_{q}, w_{r}\right\}$ and $\left\{a_{1}, b_{q}, w_{r}\right\}$, a contradiction. The second case, where the top triple is $\left\{\infty_{x}, b_{1}^{h}, b_{1}^{i}\right\}$, is similar.

In the third of the five cases, the two horizontal triples may be taken as $\left\{\infty_{x}, \infty_{y}, a_{1}^{h}\right\}$ and $\left\{\infty_{x}, a_{q}^{i}, b_{q}^{j}\right\}, q>1$. If the third (horizontal) triple is $\left\{\infty_{y}, a_{q}^{i}, b_{q}^{k}\right\}$, then the fourth is $\left\{a_{1}^{h}, b_{q}^{j}, b_{q}^{k}\right\}$ which is neither horizontal nor vertical. On the other hand, if the third (horizontal) triple is $\left\{\infty_{y}, a_{q}^{k}, b_{q}^{j}\right\}$, then the fourth is $\left\{a_{1}^{h}, a_{q}^{i}, a_{q}^{k}\right\}$ which is neither horizontal nor vertical. The fourth case, where the top triple is $\left\{\infty_{x}, \infty_{y}, b_{1}^{h}\right\}$, is similar and there only remains the possibility that the two horizontal triples are of the form $\left\{\infty_{x}, \infty_{y}, \infty_{z}\right\}$ and $\left\{\infty_{x}, a_{q}^{i}, b_{q}^{j}\right\}, q>1$.

In this case, suppose firstly that the third triple of $P$ is $\left\{\infty_{y}, a_{q}^{i}, b_{q}^{k}\right\}$. Then the fourth triple is $\left\{\infty_{z}, b_{q}^{j}, b_{q}^{k}\right\}$. However, no triple of the $\operatorname{BQFSTS}(2 n+$ $m,-m)$ is labelled $M, B, B$, and so this also gives a contradiction. Suppose secondly that the third triple of $P$ is $\left\{\infty_{y}, a_{q}^{k}, b_{q}^{j}\right\}$. Then the fourth triple is $\left\{\infty_{z}, a_{q}^{i}, a_{q}^{k}\right\}$, which contradicts the assumption that there are no triples labelled $M, A, A$.

It follows from the above argument that no point $\infty_{x} \in M$ can lie in $P$. But then, if $P$ contains two horizontal triples, they must be from the same level because they intersect. In this case, all four triples of $P$ come from a common level, again contradicting the anti-Pasch properties of the $\operatorname{STS}(2 n+m)$ and the $\operatorname{STS}(2 n+m,-m)$.

Thus $P$ can contain at most one horizontal triple, and any such triple cannot contain any $\infty_{x} \in M$. Suppose that $P$ does indeed contain exactly one such horizontal triple. At least two points of this triple will have a common projection. Furthermore, the vertical triples of $P$ through these two points must intersect in a common point. But then the third points of each of these two vertical triples must also share a common projection, and consequently these two third points must be at the same level. In this case, the fourth triple of $P$ cannot be vertical (or indeed, horizontal).

It now follows that $P$ must comprise four vertical triples and, consequently, at most two points of $P$ can come from any given level. If there were two points of $P$ at the $q$ th level, both lying in $A_{q}$ or both lying in $B_{q}$, then the other four points would have to come from precisely two levels, say levels $r$ and $s$. Also, the two points at level $r$ would both lie in $A_{r}$ or both lie in $B_{r}$, and the two points at level $s$ would both lie in $A_{s}$ or both lie in $B_{s}$. 
But then the four triples of $P$ would form a sub-TD $(3,2)$ of the $\operatorname{TD}(3, n)$, giving a contradiction. Hence, if $P$ contains a pair of points at any level, say the $q$ th, then one of these points must lie in $A_{q}$ and the other in $B_{q}$.

There are now four possibilities to consider for the vertical triples of $P$ and all of these lead to a contradiction in a similar fashion to one another. The possibilities are that $P$ contains

(a) a pair of points at each of three distinct levels, or

(b) a pair of points at each of two distinct levels with the remaining two points at a further two distinct levels, or

(c) a pair of points at one level with the remaining four points at a further four distinct levels, or

(d) six points, all at distinct levels.

In each case, projecting back to the QFSTS $(v)$ would give a Pasch configuration in that design. Thus the $\operatorname{STS}(n(v-1)+m)$ is anti-Pasch.

Remark. If a $\operatorname{QFSTS}(m)$ exists, then the existence of a $\operatorname{BQFSTS}(2 n+$ $m,-m)$ entails the existence of a $\operatorname{QFSTS}(2 n+m)$ with the $\operatorname{QFSTS}(m)$ as a subsystem. If the $\operatorname{QFSTS}(2 n+m)$ design used for the top level in the construction is formed in this way, then subsequent removal of the QFSTS $(m)$ subsystem from the $\operatorname{QFSTS}(n(v-1)+m)$ leaves a $\operatorname{BQFSTS}(n(v-1)+m,-m)$. Thus the construction provides a recursive method for the production of antiPasch $m$-bipartite designs. We make use of this observation in the final step of our proof in Section 3.

\section{The Applications}

Having established Theorem 2.1, we may put it to use in the manner demonstrated by the following example. In arithmetic set density terms, this example deals with one sixth of the remaining possible exceptions to the conjecture.

Example 3.1 From a BQFSTS $(19,-3)$ we may construct a QFSTS $(144 t+$ 67) for $t \geq 0$, by applying Theorem 2.1, with $v=18 t+9, n=8$ and $m=3$, so that $n(v-1)+m=144 t+67$. 
Recall that a $\operatorname{QFSTS}(v)$ is known to exist for all $v \equiv 3(\bmod 6)$ and for $v=19$. A BQFSTS $(19,-3)$ may be constructed as follows. Take a QFSTS(19) and choose a triple of the system; the points of the triple form the points of $M$. Define a graph whose vertices are the 16 remaining points and such that two vertices are adjacent if they appear in a triple together with a point of $M$. Clearly a $\operatorname{BQFSTS}(19,-3)$ may be obtained from the QFSTS(19) if and only if this graph is bipartite. There are four non-isomorphic cyclic STS(19)s of which two are anti-Pasch [5]; these are denoted by A2 and A4, the latter being the so-called Netto system. Because of the cyclic nature of these systems, there are in each case only three inequivalent possibilities for the triple to form $M$, namely a triple from each of the three orbits forming the system. We find that the Netto system does not give a BQFSTS $(19,-3)$, but the system A2 does. This system is generated under the action of the mapping $i: \rightarrow i+1(\bmod 19)$ from the starter blocks $\{0,1,4\},\{0,2,12\}$ and $\{0,5,13\}$. We may choose $M=\{0,1,4\}$, $A=\{2,6,7,8,13,15,17,18\}$ and $B=\{3,5,9,10,11,12,14,16\}$.

In fact it is possible to use Theorem 2.1, together with other results and induction, to reduce the proof of the anti-Pasch conjecture to the construction of a small number of $m$-bipartite designs. Recall from the Introduction that a $\operatorname{QFSTS}(w)$ is known to exist for all admissible $w<100$ (apart from $w=7$ or 13), and for all admissible $w>100$ except for the classes $w \equiv 13,31$ or 67 $(\bmod 72)$.

In the Tables below, the existence of an appropriate QFSTS $(v)$ is guaranteed by results cited in the Introduction, or by earlier entries in the Tables themselves, or by use of an inductive argument. The Tables and associated Notes should be read sequentially. References to earlier entries in the Tables and to the use of an inductive argument are explained in the associated Notes. We remark that alternative strategies may be possible, we have selected the strategy which seems to us to offer the maximum gain at minimum cost.

The class $w \equiv 31(\bmod 72)$ may be covered as shown in Table 1 by writing $w=n(v-1)+m$. 


\begin{tabular}{|c|c|c|c|l|l|}
\hline$w(\bmod 72)$ & $v$ & $n$ & $m$ & $\begin{array}{l}\text { m-bipartite } \\
\text { designs required }\end{array}$ & Comment \\
\hline 31 & $\begin{array}{c}6 t+3 \\
(t \geq 0)\end{array}$ & 12 & 7 & BQFSTS $(31,-7)$ & $\begin{array}{l}\text { This design is } \\
\text { given in Section 4 }\end{array}$ \\
\hline
\end{tabular}

Table 1: $w \equiv 31(\bmod 72)$

The class $w \equiv 13(\bmod 72)$ may be split into the subclasses $w \equiv 13,85$ or 157 ( $\bmod 216)$. These may then be covered as shown in Table 2 by writing $w=n(v-1)+m$. The designs indicated by an $\exists$ are available from the JCD website [15].

\begin{tabular}{|c|c|c|c|l|l|}
\hline$w(\bmod 216)$ & $v$ & $n$ & $m$ & $\begin{array}{l}\text { m-bipartite } \\
\text { designs required }\end{array}$ & Comment \\
\hline 85 & $\begin{array}{c}6 t+3 \\
(t \geq 0)\end{array}$ & 36 & 13 & BQFSTS $(85,-13)$ & $\exists$ \\
\hline 157 & $\begin{array}{c}12 t+9 \\
(t \geq 0)\end{array}$ & 18 & 13 & BQFSTS $(49,-13)$ & $\exists$ \\
\hline 13 & $\begin{array}{l}12 t+1 \\
(t \geq 2)\end{array}$ & 18 & 13 & $\begin{array}{l}\text { BQFSTS }(49,-13) \\
\text { BQFSTS }(67,-13)\end{array}$ & $\begin{array}{l}\text { See Note } 3.1 \\
\exists\end{array}$ \\
\hline
\end{tabular}

Table $2: w \equiv 13(\bmod 72)$

Note 3.1. The case $w \equiv 13(\bmod 216)$ requires an induction argument. We observe that there exists a QFSTS(49) and a BQFSTS(49, -13), and that the cases $w \equiv 85$ and $157(\bmod 216)$ are already established. Then combining these together with cases covered by Theorem 6.1 and Theorem 6.2 of [9], a QFSTS $(12 t+1)$ is known to exist apart from when $t$ has the form $t=18 \mathrm{~s}+1$, for $s=0,1,2, \ldots$. Suppose, inductively, that the cases $s=1,2, \ldots, s^{*}$ have already been established. Then a $\operatorname{QFSTS}(12 t+1)$ would be known to exist for all values of $t$, apart from $t=1$, up to and including the case when $12 t+1=216 s^{*}+13$. But $216 s^{*}+13>12 s^{*}+13>13$, and so there exists a QFSTS $\left(12 s^{*}+13\right)$. Applying Theorem 2.1, we could then deduce that there exists a $\operatorname{QFSTS}\left(18\left(12 s^{*}+12\right)+13\right)$, i.e. a $\operatorname{QFSTS}(12 t+1)$ for $t=18\left(s^{*}+1\right)+1$. To start the inductive argument, a QFSTS(229) is required. This design may be formed using Theorem 2.1 with $m=13, n=27$ and $v=9$; this requires a QFSTS(67) which is known to exist, and a $\operatorname{BQFSTS}(67,-13)$ which has been constructed by the authors and is available from the JCD website [15]. 
The class $w \equiv 67(\bmod 72)$ appears to require considerable subdivision. We firstly split it into two cases $w \equiv 67$ or $139(\bmod 144)$. The former case is covered in Example 3.1. The latter case may be split into five cases $w \equiv 139,283,427,571$ or $715(\bmod 720)$ and the last of these cases is further split into seven cases modulo 5040. Table 3 gives the details, again taking $w=n(v-1)+m$. The designs indicated by an $\exists$ are available from the JCD website [15]. The case $w \equiv 3595(\bmod 5040)$ is significantly more complicated than the other cases and a full explanation of this case is given in Note 3.8.

\begin{tabular}{|c|c|c|c|l|l|}
\hline$w$ & $v$ & $n$ & $m$ & $\begin{array}{l}\text { m-bipartite } \\
\text { designs required }\end{array}$ & Comment \\
\hline $67(\bmod 144)$ & $\begin{array}{c}18 t+9 \\
(t \geq 0)\end{array}$ & 8 & 3 & BQFSTS $(19,-3)$ & $\begin{array}{l}\text { Given in } \\
\text { Example 3.1 }\end{array}$ \\
\hline \hline $139(\bmod 720)$ & $\begin{array}{c}12 t+3 \\
(t \geq 0)\end{array}$ & 60 & 19 & BQFSTS $(139,-19)$ & $\begin{array}{l}\exists \\
\text { See Note 3.2 }\end{array}$ \\
\hline $283(\bmod 720)$ & $\begin{array}{c}36 t+15 \\
(t \geq 0)\end{array}$ & 20 & 3 & BQFSTS $(43,-3)$ & $\exists$ \\
\hline $427(\bmod 720)$ & $\begin{array}{c}24 t+15 \\
(t \geq 0)\end{array}$ & 30 & 7 & BQFSTS $(67,-7)$ & $\exists$ \\
\hline $571(\bmod 720)$ & - & - & - & - & $\begin{array}{l}\text { Covered by }[9] \\
\text { See Note 3.3 }\end{array}$ \\
\hline \hline $715(\bmod 5040)$ & - & - & - & - & $\begin{array}{l}\text { Covered by [9] } \\
\text { See Note 3.4 }\end{array}$ \\
\hline $1435(\bmod 5040)$ & $\begin{array}{c}240 t+69 \\
(t \geq 0)\end{array}$ & 21 & 7 & BQFSTS $(49,-7)$ & $\exists$ \\
\hline $2155(\bmod 5040)$ & $\begin{array}{c}240 t+103 \\
(t \geq 0)\end{array}$ & 21 & 13 & BQFSTS $(55,-13)$ & $\begin{array}{l}\exists \\
\text { See Note 3.5 }\end{array}$ \\
\hline $2875(\bmod 5040)$ & $\begin{array}{c}180 t+103 \\
(t \geq 0)\end{array}$ & 28 & 19 & BQFSTS $(75,-19)$ & $\begin{array}{l}\exists \\
\text { See Note 3.6 }\end{array}$ \\
\hline $4315(\bmod 5040)$ & $\begin{array}{c}360 t+309 \\
(t \geq 0)\end{array}$ & 14 & 3 & BQFSTS $(31,-3)$ & $\exists$ \\
\hline $5035(\bmod 5040)$ & - & - & - & - & $\begin{array}{l}\text { Covered by [9] } \\
\text { See Note 3.7 }\end{array}$ \\
\hline $3595(\bmod 5040)$ & - & - & - & BQFSTS $(61,-19)$ & $\begin{array}{l}\exists \\
\text { See Note 3.8 }\end{array}$ \\
\hline \hline
\end{tabular}

Table 3: $w \equiv 67(\bmod 72)$

Note 3.2. The existence of a BQFSTS $(139,-19)$ entails that of a QFSTS(139), since a QFSTS(19) is known. 
Note 3.3. If $w \equiv 571(\bmod 720)$, then we may employ Theorem 2.4 of $[9]$. Take a QFSTS(15) and a QFSTS $(48 t+39)(t \geq 0)$ to form a QFSTS $(15(48 t+$ $38)+1)$, i.e. a $\operatorname{QFSTS}(720 t+571)$.

Note 3.4. If $w \equiv 715(\bmod 5040)$, then we may employ Theorem 2.4 of $[9]$. Take a QFSTS $(360 t+51)(t \geq 0)$ and a QFSTS $(15)$ to form a QFSTS $((360 t+$ $51) \cdot 14+1)$, i.e. a $\operatorname{QFSTS}(5040 t+715)$.

Note 3.5. The number $v=240 t+103(t \geq 0)$ lies in one of the residue classes 103, 343 or 583 modulo 720, and hence in one of the residue classes 31,55 or 7 modulo 72. It follows from results cited in the Introduction and from Table 1, that a $\operatorname{QFSTS}(v)$ exists.

Note 3.6. The number $v=180 t+103(t \geq 0)$ lies in one of the residue classes 103, 283, 463 or 643 modulo 720, and hence in one of the residue classes $31(\bmod 72), 283(\bmod 720)$ or $67(\bmod 144)$, all of which correspond to earlier entries in the Tables. Hence a QFSTS $(v)$ exists.

Note 3.7. If $w \equiv 5035(\bmod 5040)$, then we may employ Theorem 4.1 and Theorem 6.1 of [9]. From the latter theorem, there exists a QFSTS(720t+ $721)$ for $t \geq 0$. Take a $\operatorname{QFSTS}(9)$ and a $\operatorname{QFSTS}(720 t+721)$ to form a $\operatorname{QFSTS}(7(720 t+719)+2)$, i.e. a $\operatorname{QFSTS}(5040 t+5035)$.

Note 3.8. Following earlier entries in the Tables, the only residue class for which the conjecture remains unsettled is $v \equiv 3595(\bmod 5040)$. This is dealt with as follows.

Since $43 \not \equiv 3595(\bmod 60)$, it is already established that a $\operatorname{QFSTS}(60 t+$ 43) exists for $t \geq 0$. We have constructed a $\operatorname{BQFSTS}(61,-19)$ and this is available from the JCD website [15]. Recalling the Remark following the proof of Theorem 2.1, it follows that a BQFSTS $(21(60 t+42)+19,-19)$, i.e. a BQFSTS $(1260 t+901,-19)$, exists for $t \geq 0$. Addition of a $\operatorname{BQFSTS}(19,-3)$ (given in Example 3.1) to such a design produces a BQFSTS $(1260 t+901,-3)$. Reapplying our construction with $v=9, n=630 t+449$ and $m=3$ gives a $\operatorname{QFSTS}((630 t+449) .8+3)$, i.e. a QFSTS $(5040 t+3595)$.

We conclude this Section by summarising the result of our Tables and formally stating our main result as a Theorem.

Theorem 3.1 If $v \equiv 1$ or $3(\bmod 6)$ and $v \neq 7$ or 13 , then there exists a $\operatorname{QFSTS}(v)$. 


\section{The auxiliary designs}

A full listing of the triples of a $\operatorname{BQFSTS}(31,-7)$ is given below. For clarity, we list blocks omitting set brackets and commas. A specimen of each of the BQFSTS $(u,-m)$ designs used in this paper is available from the JCD website [15]. These correspond to $(u, m)=(19,3),(31,3),(31,7),(43,3)$, $(49,7),(49,13),(55,13),(61,19),(67,7),(67,13),(75,19),(85,13)$ and $(139,19)$. We use the convention that the point set is $\{1,2, \ldots, u\}$ with partition $M=$ $\{1,2, \ldots, m\}, A=\{m+1, m+2, \ldots, m+n\}$ and $B=\{m+n+1, m+n+$ $2, \ldots, m+2 n\}$, so that $u=m+2 n$.

In each case, the design was obtained by a modified hill-climbing algorithm, of the type described in [13]. The principal modifications used were to start with a fixed set of triples. Except for the parameter pair $(61,19)$ these were those triples containing the points of $M$, and this thereby ensured that any system produced would have the necessary $m$-bipartite property. There are many ways to select these initial triples. At each subsequent stage of the hill-climbing algorithm, a check was carried out to ensure that any additional or replacement triple did not result in the formation of a Pasch configuration. In the case of the pair $(61,19)$, the parameters are rather tight and it was found to be more effective to start the hill-climbing algorithm with a fixed set of triples covering all $A, A$ and $B, B$ pairs. Again this ensured that any system produced would be 19-bipartite.

$\begin{array}{rrr}1 & 8 & 20 \\ 1 & 15 & 31 \\ 2 & 10 & 20 \\ 2 & 17 & 29 \\ 3 & 12 & 20 \\ 3 & 19 & 27 \\ 4 & 14 & 26 \\ 5 & 9 & 27 \\ 5 & 16 & 26 \\ 6 & 11 & 31 \\ 6 & 18 & 26 \\ 7 & 13 & 23 \\ 8 & 9 & 16 \\ 8 & 27 & 28 \\ 9 & 20 & 22 \\ 11 & 13 & 20 \\ 12 & 17 & 28 \\ 13 & 19 & 25 \\ 16 & 22 & 29 \\ 20 & 28 & 29 \\ 22 & 28 & 30 \\ 27 & 30 & 31\end{array}$

$\begin{array}{rrr}1 & 9 & 30 \\ 1 & 16 & 23 \\ 2 & 11 & 28 \\ 2 & 18 & 23 \\ 3 & 13 & 26 \\ 4 & 8 & 21 \\ 4 & 15 & 24 \\ 5 & 10 & 21 \\ 5 & 17 & 22 \\ 6 & 12 & 21 \\ 6 & 19 & 20 \\ 7 & 14 & 24 \\ 8 & 10 & 13 \\ 9 & 10 & 17 \\ 10 & 11 & 12 \\ 11 & 15 & 16 \\ 12 & 18 & 31 \\ 14 & 16 & 17 \\ 17 & 21 & 30 \\ 21 & 22 & 24 \\ 23 & 24 & 26 \\ & & \end{array}$

$\begin{array}{rrr}1 & 10 & 22 \\ 1 & 17 & 27 \\ 2 & 12 & 22 \\ 2 & 19 & 31 \\ 3 & 14 & 23 \\ 4 & 9 & 31 \\ 4 & 16 & 28 \\ 5 & 11 & 29 \\ 5 & 18 & 28 \\ 6 & 13 & 27 \\ 7 & 8 & 31 \\ 7 & 15 & 30 \\ 8 & 11 & 14 \\ 9 & 11 & 24 \\ 10 & 14 & 19 \\ 11 & 17 & 23 \\ 12 & 26 & 27 \\ 14 & 20 & 27 \\ 18 & 20 & 24 \\ 21 & 23 & 28 \\ 23 & 25 & 29\end{array}$

$\begin{array}{rrrrrr}\text { BQFSTS }(31,-7) \\ 1 & 11 & 26 & 1 & 12 & 24 \\ 1 & 18 & 25 & 1 & 19 & 29 \\ 2 & 13 & 30 & 2 & 14 & 25 \\ 3 & 8 & 22 & 3 & 9 & 28 \\ 3 & 15 & 29 & 3 & 16 & 25 \\ 4 & 10 & 23 & 4 & 11 & 27 \\ 4 & 17 & 20 & 4 & 18 & 30 \\ 5 & 12 & 23 & 5 & 13 & 31 \\ 5 & 19 & 24 & 6 & 8 & 23 \\ 6 & 14 & 28 & 6 & 15 & 22 \\ 7 & 9 & 25 & 7 & 10 & 27 \\ 7 & 16 & 20 & 7 & 17 & 26 \\ 8 & 12 & 30 & 8 & 15 & 18 \\ 9 & 12 & 13 & 9 & 14 & 18 \\ 10 & 15 & 28 & 10 & 16 & 31 \\ 11 & 18 & 19 & 11 & 22 & 25 \\ 13 & 14 & 22 & 13 & 15 & 21 \\ 14 & 29 & 31 & 15 & 17 & 25 \\ 19 & 23 & 30 & 20 & 21 & 26 \\ 21 & 25 & 31 & 21 & 27 & 29 \\ 24 & 25 & 27 & 24 & 28 & 31\end{array}$

$\begin{array}{rrr}1 & 13 & 28 \\ 2 & 8 & 24 \\ 2 & 15 & 27 \\ 3 & 10 & 24 \\ 3 & 17 & 31 \\ 4 & 12 & 25 \\ 4 & 19 & 22 \\ 5 & 14 & 30 \\ 6 & 9 & 29 \\ 6 & 16 & 30 \\ 7 & 11 & 21 \\ 7 & 18 & 22 \\ 8 & 17 & 19 \\ 9 & 15 & 23 \\ 10 & 18 & 29 \\ 12 & 14 & 15 \\ 13 & 16 & 24 \\ 15 & 19 & 26 \\ 20 & 23 & 31 \\ 22 & 23 & 27 \\ 24 & 29 & 30\end{array}$

$1 \quad 14 \quad 21$

$\begin{array}{lll}2 & 9 & 26\end{array}$

$\begin{array}{lll}2 & 16 & 21\end{array}$

$\begin{array}{lll}3 & 11 & 30\end{array}$

$\begin{array}{lll}3 & 18 & 21\end{array}$

$\begin{array}{lll}4 & 13 & 29\end{array}$

$\begin{array}{lll}5 & 8 & 25\end{array}$

$\begin{array}{lll}5 & 15 & 20\end{array}$

$\begin{array}{lll}6 & 10 & 25\end{array}$

$\begin{array}{lll}6 & 17 & 24\end{array}$

$\begin{array}{lll}7 & 12 & 29\end{array}$

$\begin{array}{lll}7 & 19 & 28\end{array}$

$\begin{array}{lll}8 & 26 & 29\end{array}$

$\begin{array}{lll}9 & 19 & 21\end{array}$

$\begin{array}{lll}10 & 26 & 30\end{array}$

$\begin{array}{lll}12 & 16 & 19\end{array}$

$\begin{array}{lll}13 & 17 & 18\end{array}$

$\begin{array}{lll}16 & 18 & 27\end{array}$

$\begin{array}{lll}20 & 25 & 30\end{array}$

$\begin{array}{lll}22 & 26 & 31\end{array}$

$\begin{array}{lll}25 & 26 & 28\end{array}$ 


\section{Acknowledgements.}

We thank Niall Adams of Imperial College and Matt Bernstein of Goldsmiths College for their substantial assistance in running searches for some of the auxiliary designs on a variety of computing equipment. We also thank Charlie Colbourn of the University of Vermont for a simplification of the main construction, and we thank the referees for several helpful comments which have improved the presentation of our results. Finally, we thank Jeff Dinitz of the University of Vermont for his help in getting the auxiliary designs onto the JCD website.

\section{References}

[1] A.E. Brouwer, Steiner triple systems without forbidden subconfigurations, Mathematisch Centrum Amsterdam, ZW, (1977), 104/77.

[2] C.J. Colbourn, E. Mendelsohn, A. Rosa and J. Siráň, Anti-mitre Steiner triple systems, Graphs and Combinatorics 10, (1994), 215-224.

[3] C.J. Colbourn, J.H. Dinitz and D.R. Stinson, Combinatorial designs in communications, Surveys in Combinatorics, 1999 (LMS Lecture Note Series, 267), Cambridge University Press, (1999), 37-100.

[4] P. Erdös, Problems and results in combinatorial analysis, Creation in Mathematics 9, (1976), 25.

[5] T.S. Griggs and J.P. Murphy, 101 Anti-Pasch Steiner triple systems of order 19, J. Combin. Math. Combin. Comput. 13, (1993), 129-141.

[6] T.S. Griggs, J. Murphy and J.S. Phelan, Anti-Pasch Steiner triple systems, J. Comb. Inf. Syst. Sci. 15, (1990), 79-84.

[7] A. Kotzig, C.C. Lindner and A. Rosa, Latin Squares with no subsquares of order two and disjoint Steiner triple systems, Utilitas Math. 7, (1975), 287-294.

[8] A. Kotzig and J. Turgeon, On certain constructions for Latin squares with no Latin subsquares of order two, Discrete Math. 16, (1975), 263270 . 
[9] A.C.H. Ling, C.J. Colbourn, M.J. Grannell and T.S. Griggs, Construction techniques for anti-Pasch Steiner triple systems, J. London Math. Soc., to appear.

[10] R. Mathon, K.T. Phelps and A. Rosa, Small Steiner triple systems and their properties, Ars Comb. 15, (1983), 3-110.

[11] M. McLeish, On the existence of Latin Squares with no subsquares of order two, Utilitas Math. 8, (1975), 41-53.

[12] J.P. Murphy, Steiner triple systems and cycle structure, Ph.D. thesis, University of Central Lancashire, (1999).

[13] D.R. Stinson, Hill-climbing algorithms for the construction of combinatorial designs, Ann. Discrete Math. 26, (1985), 321-334.

[14] D.R. Stinson and H. Ferch, 2000000 Steiner triple systems of order 19, Math. Comput. 44, (1985), 533-535.

[15] Journal of Combinatorial Designs website. 